\title{
Retos y tendencias sobre el futuro de la investigación acerca del aprendizaje con tecnologías digitales.
}

\author{
Challenges and future trends of research on learning using digital \\ technologies. \\ Begoña Gros \\ Universidad de Barcelona. Barcelona. España. \\ bgros@ub.edu
}

\section{Resumen}

El objetivo de este artículo es analizar los retos y tendencias de la investigación acerca del aprendizaje con tecnologías digitales.En las dos últimas décadas, las investigaciones sobre el uso de las tecnologías digitales para la formación y el aprendizajese ha consolidado como un ámbito de investigaciónmultidisciplinar. Los resultados obtenidos muestran algunas limitaciones ya que no proporcionan respuestas suficientes que permitan analizar y diseñar las prestaciones tecno-pedagógicas adecuadas para favorecer el aprendizaje. A menudo, las investigaciones seplantean en términos comparativos tratando de determinar los beneficios de una determinada tecnología en el aprendizaje utilizando modelos causa-efecto que simplifican realidades en las que intervienen muchas variables.

Creemos que es el momento de reflexionar sobre la utilización de nuevas metodologías e instrumentos de investigación que puedan mejorar la calidad y los resultados de las investigaciones.En el artículo se analiza la formulación de las preguntas de investigación que son claves para diseñar los estudios. Los métodos de investigación utilizados y el uso de infraestructuras tecnológicas para la obtención masiva de datos. Por último, se analizar la importancia de compartir datos y resultados a partir del uso de repositorios para la difusión del conocimiento.

\section{Palabras clave}

Investigación sobre TICs y educación, investigación basada en el diseño, e-investigación, depósitos digitales.

\begin{abstract}
The aim of this article is to analyse the challenges and trends in research on learning with digital technologies. In the last two decades, research on the use of digital technologies for education and learning has become an area of multidisciplinary research. The results show some limitations because it does not provide enough responses to analyse and design the appropriate teaching technological features to enhance learning. Often, research is trying to determine the benefits of a technology in learning using models that simplify reality. We believe it is time to consider the use of new methodologies and research tools that can improve the quality and results of investigations. The article discusses the formulation of research questions that are key to designing the studies. We describe the main research methods used and the use of technological infrastructure for the massive data collection. Finally, we analyse the importance of sharing data and results through the use of repositories for the dissemination of knowledge.
\end{abstract}

Key words:

Research on ICTs and education, research-based design, e-research, digital repositories. 


\section{Introducción}

El objetivo principal de este artículo es analizar la situación actual de la investigación sobre el uso de las TICs en la educación y apuntar las tendencias y retos futuros. En las dos últimas décadas, el número de trabajos se han incrementado enormemente (Hung, 2012).Es un ámbito consolidado de investigación con publicaciones reconocidas por la comunidad internacional. No obstante, los resultados obtenidos muestran algunas limitaciones. En general, tenemos instrumentos adecuados para aproximarnos a los análisis macro que nos proporcionan datos sobre uso e integración de las TICsen los centros escolares, universidades, etc. Sin embargo, cuando pasamos al análisis meso y micro nos encontramos con muchas limitaciones debido a la escasez de trabajos que proporcionan datos a lo largo del tiempo y al tipo de metodologías utilizadas.

Los estudios sobre el uso de las TICs para el aprendizaje forma parte de un ámbito de estudio amplio y multidisciplinar en el que se combinan miradas e intereses muy diversos. No obstante, muchas de las investigaciones comparten un mismo problema: no proporcionan respuestas suficientes que permitan analizar y diseñar las prestaciones tecno-pedagógicas adecuadas para favorecer el aprendizaje. Además, tampoco se aprecia una investigación acumulativa y, a menudo, se tiene la sensación de estar siempre formulando las mismas preguntas.

Después de treinta años de investigación, todavía hay una ingenua suposición de que la tecnología por sí misma mejora el aprendizaje. Por ello, es frecuente encontrar trabajos sobre las TICs en educaciónsustentados sobre un enfoque muy ingenuo que considera que la tecnología transforma las prácticas educativas. Tambiénhay investigadores que se sitúan en el otro extremo y creen que la tecnología es una simple"herramienta" que facilita ciertas prácticas pero que lo importante es la metodología. En estos casos, incluso se intenta mostrar como no hay nada nuevo ya que en realidad estamos haciendo lo mismo pero con soportes diferentes. En mi opinión, ambos enfoques son limitados. Cuando las tecnologías y los diseños educativos se comunican hayuna interdependencia dinámica y como consecuencia emergen prácticas que no siempre podemos predecir. Además, no todos los cambios metodológicos y tecnológicos tienen los mismos niveles de impacto. De este modo, no es comparable la transformación cognitiva que supone el uso de la tecnología en la producción escrita que un programa para aprender las capitales europeas. Hay tecnologías y metodologías que modifican practicas y formas de aprendizaje de forma profunda mientras que otras mejoran algunas dinámicas o procesos sin incidir en cambios profundos. Por consiguiente, la aproximación al análisis y estudio no puede ser el mismo ni fundamentarse únicamente, como sucede muy frecuentemente, en las percepciones de los participantes.

Otro problema importante lo constituyela formulación de las preguntas de investigación. En muchos casos, éstas se plantean en términos comparativos tratando de determinar los beneficios de una determinada tecnología en el aprendizaje utilizando modelos causaefecto que simplifican realidades en las que intervienen muchas variables.

El terreno no es homogéneo y Hung (2012) apunta quelos países más prolíficos en materia de investigación en el uso de las TICs (Inglaterra, Australia, Alemania, Italia, España, Japón) para la educación, en lugar de centrarse en los aspectos técnicos, están

Retos y tendencias sobre el futuro de la investigación acerca del aprendizaje con tecnologías digitales. Begoña Gros. 
cada vez más preocupados por el aspecto educativo. Por el contrario, la mayoría de trabajos publicados por autores asiáticosestán muy orientados a los aspectos tecnológicosa excepción de losautores taiwanesesque estudianambos puntos de vista.

El aumento de la masa crítica de investigadores es un aspecto muy importante que puede beneficiar al incremento de conocimiento. Creemos que es el momento dereflexionar sobre la utilización de nuevas metodologías e instrumentos de investigación que puedan mejorar la calidad y los resultados de las investigaciones.

El objetivo general de este artículoes analizar los retos y tendencias de la investigación acerca del aprendizaje con tecnologías digitales. Para ello, analizaremos la formulación de las preguntas de investigación que son claves para diseñar los estudios. En segundo lugar, nos centraremos en los métodos de investigación con especial hincapié en la investigación basada en el diseño. En tercer lugar, planteamos el problema de la obtención de datos y posibles soluciones a través del uso de infraestructuras tecnológicas y, finalmente, analizamos la implicación de la publicación en abierto para la difusión del conocimiento.

\section{Las preguntas de investigación}

Un problema importante del enfoque y la orientación de muchos estudios e investigaciones es que tratan de responder a preguntassobre los beneficios del uso de la tecnología para el aprendizaje.Es frecuente encontrar investigaciones centradas en el análisis delas consecuencias de incorporar una determinada tecnología a partir de estudios basados en experimentos o en prácticas aplicadas en un tiempo muy breve. La pregunta típica es "¿cuál es el efecto de "x" en el aprendizaje?", ¿se aprende mejor cuando se utiliza "x?. Normalmente, "x" es alguna software o tecnología específica. Si revisamos los estudios en la década de 1980, 1990, y 2000, las diferencias están en el contenido de la "x" (multimedia / wiki / blog / realidad aumentada, ordenadores, tabletas, móviles, etc.). Estamos cambiando las preguntas en función de la evolución de la tecnología. Hay una repetición constante de la misma pregunta cambiando la tecnología concreta. Lo más fascinante es que este tipo de estudios siempre tienen un final feliz: los alumnos están mas motivados.

Uno de los problemas más importantes de la investigación sobre las TICs es la formulación de las preguntas. No se trata de saber sí se aprende mejor sino qué se aprende y lo que realmente interesa es comprender el cómo. En otras palabras, necesitamos entender cómo diseñar entornos y situaciones educativas que puedan mejorar el aprendizaje.La tecnología como tal no determina la naturaleza de su aplicación, pero co-evoluciona con la transformación gradual de las prácticas. No es una simple adaptación, sino un proceso creativo en el que recíprocamente las herramientas facilitan las prácticas y las prácticas innovadoras se crean con el fin de hacer un mejor uso de las nuevas posibilidades que ofrecen las tecnologías. Es importante entender esta relación dialéctica.Elaborar conocimiento sobre el usode las tecnologías digitales en el aprendizajesuponeentrar en un terreno de diálogo constante entre los diseños tecnológicos y pedagógicos.

En un artículo sobre los problemas generales de la investigación en educación, Reeves (2011) crítica que en muchas investigaciones la principal obsesión es la rigurosidad

Retos y tendencias sobre el futuro de la investigación acerca del aprendizaje con tecnologías digitales. Begoña Gros. 
metodológica con el objetivo de que el trabajo sea publicado. No obstante, se pierde de vista la relevancia del estudio y así es como podemos encontrar artículos con diseños metodológicos perfectos pero con preguntas totalmente irrelevantes que no aportan ningún valor a la teoría y/o a la práctica educativa.

Reimann (2009) afirma que mientras las preguntas de investigación involucren relaciones entre variables no podremos avanzar en el análisis de investigaciones que traten de explicar los cambios y procesos. Según este autor, es preciso conceptualizar los procesos de desarrollo y de cambio como una secuencia de eventos que tienen unidad y son coherentes con el tiempo. Un evento es una especie de "actor", pero el "actor" no tiene que ser una persona, sino que puede ser un grupo, una organización, una tecnología, una metodología, etc.

En definitiva, es preciso incorporar preguntas que orienten al investigación hacia el análisis de redes y que permitan comprender ecologías de aprendizaje. Esta aproximación obliga a avanzar en la utilización de nuevos métodos e instrumentos de investigación.

\section{Los métodos de investigación}

Zawacki-Richte and Bäcker (2009) realizaron un análisis de las publicaciones sobre elearning entreel 2000 y 2008 para describer el estado de la investigación. Loss resultados rebelan un fuerte desequilibrio; las investigaciones desde la perspectiva micro son las más numerosas mientras que las centradas en análisis meso o macro son mucho más escasas. En más del 50\% de los artículos publicados, los temas más frecuentes son: interaction y communication (17.6\%), diseño instructivo (17.4\%), y characteristics de los estudiantes $(16.3 \%)$.

Los estudios micro aportan datos que provienen de experiencias que se han llevado a cabo enpocas semanas (Zawacki-Richte and Bäcker; 2009). Este aspecto también lo han apuntado Cabero y su equipo (2009) como resultado del meta-análisis realizado en España. Un 65,4\% de los estudios están basados en análisis de experiencias de corta duración y solo un 28,4\% tienen una duración algo superior. Chin (2007) en un análisis centrado en las publicaciones sobre CSCL (aprendizaje colaborativo con soporte tecnológico)entre el 2005 y el 2007 afirma que casi un 50\% de los estudios analiza grupos de estudiantes trabajando juntos durante menos de un mes. Tenemos un problema importante ya que hay falta de análisis de los cambios a lo largo del tiempo y análisis muy parciales de la realidad.

En relación a las metodologías de investigación, el estudio de Cabero et al. (2009) señala que un 48,2\% de las investigaciones son descriptivas, un 20,6\% experimentales y $31,3 \%$ son estudios de caso. En las investigaciones micro se suelen aplicar métodos experimentales cuando se analizan determinados desarrollos tecnológicos y se comparan los resultados entre el grupo experimental y de control. Cuando la investigación micro tiene una aproximación más metodológica, la aproximación suele ser más cualitativa. El problema, en ambos casos, es la escasa orientación hacia la mejora de la práctica educativa.

Retos y tendencias sobre el futuro de la investigación acerca del aprendizaje con tecnologías digitales. Begoña Gros. 
El transito de un modelo educativo transmisivo a un modelo educativo centrado en las actividades pone de relieve la necesidad de plantear investigaciones mucho más holísticas centradas en aspectos relacionados con el diseño del entornos de aprendizaje y las prestaciones tecnológicas para incrementar el aprendizaje. Cuban (2001) considera que las culturas de investigación educativa deben ser próximas a los procesos de diseño. Y, en los últimos años, se ha empezado a utilizar la investigación basada en el diseño en las investigaciones que intentan dar cuenta de los cambios y los procesos innovadoressituadas a un nivel micro y meso.

Este tipo de investigación recibe diferentes nombres; el diseño de experimentos (Brown, 1992, Collis, 1992), la investigación formativa (Newman, 1990), la investigación basada en el diseño educativo (Akker, et al. 2006). En el artículo publicado por el autodenominado DBR (The Design-Based Research Collective, 2003) se afirma que este enfoque permite "el estudio del aprendizaje en contexto a través del diseño sistemático y del estudio de las estrategias y herramientas instruccionales. La investigación basada en el diseño puede ayudar a crear y ampliar el conocimiento acerca del desarrollo, la puesta en marcha y la sostenibilidad de ambientes de aprendizaje innovadores" (DBR, 2003). Es "investigación en, dentro de y sobre el diseño" (Rowland, 2008).

"La investigación basada en el diseño investiga características del diseño, no sólo nuevos modelos formativos, permite identificar qué características del diseño resultan más eficaces que otras con respecto a un determinado resultado, y por qué" (Reeves, 2011:13). Este tipo de investigación se lleva a cabo en entornos de aprendizaje reales y complejos y en presencia de diversidad de variables que no siempre pueden ser controladas ni determinadas a priori. Por esta razón, la determinación de los elementos que pueden estar afectando el éxito del diseño es uno de los aspectos críticos. Éstos se detectan a partir de la identificación y análisis de las contradicciones o conflictos que se presentan durante el desarrollo de las actividades poniendo en evidencia los aspectos que pueden estar afectando el éxito o fracaso.

En el diseño de la experimentación es importante tener presente: las condiciones locales del contexto de intervención, la revisión constante a partir del diseño y la experimentación, a relación entre múltiples variables dependientes, el estudio de las interacciones sociales de los participantes y la coparticipación en el diseño y análisis de los resultados. 


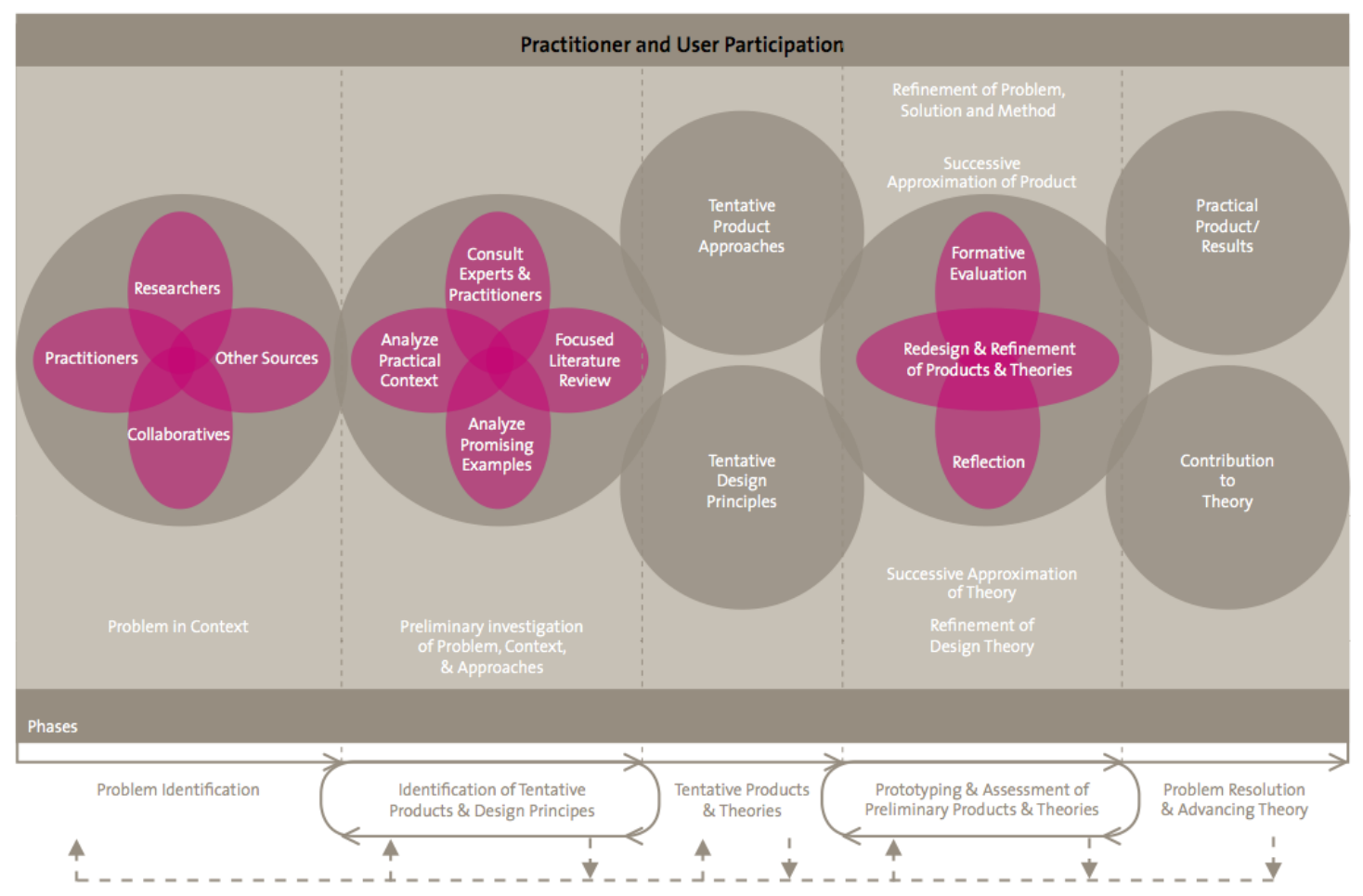

Gráfico 1. Fuente: Bannan-Ritland (2003).

Hay propuestas diversas sobre como poner en practica este tipo de metodología pero todos los autores coinciden en apuntar las fases más importantes (gráfico 1). En la fase preliminar se analizan las necesidades y el contexto de análisis, la revisión de los estudios previos sobre el tema y se elabora el marco teórico de referencia. En la segunda fase, se diseña la propuesta. La fase de iteración consiste en aplicar el experimento y refinar la propuesta a través de un sistema de 3 o 4 repeticiones que permitan mejorar y perfeccionar la intervención. En síntesis, se diseña una intervención muy fundamentada desde el punto de vista teórico, se aplica, se analizan los resultados y se hace una modificación que vuelve a ser aplicada y revisada hasta que se considera que ya no se pueden obtener más datos relevantes. Es un proceso iterativo y cíclico que puede ser replicado en diferentes situaciones.

La aplicación de esta metodología combina instrumentos diversos en función de los objetivos de la investigación. Lo importante es aportar una mejora en el conocimiento teórico y práctico. A pesar de las bondades de este planteamiento también se han señalado limitaciones importantes. Reimann (2011) sostiene que a pesar del planteamiento iterativo se suelen hacer pocas iteraciones y, en el análisis, se mantiene una aproximación causal de la investigación lo que dificulta hacer escalable los resultados. Goodyear (2011) considera que es una metodología interesante pero que todavía no ha aportado conocimiento sobre el propio diseño y lo que significa analizar un sistema e interpretar la realidad como un ecología de aprendizaje. 


\section{La e-research}

Recientemente se ha empezado a utilizar el terminoe-research de forma genérica al tratar de las nuevas metodologías de investigación derivadas del uso de Internet. Las tecnologías actuales permiten integrar diversas fuentes de datos y manejar técnicas que contribuyen a obtener nuevos conocimientos. Existe un gran potencial en la investigación haciendo uso de la red ya que ésta permite la posibilidad de compartir, integrar y acceder a datos masivos. Los resultados empiezan a ser evidentes en algunos científicos en los que ya hace años se han creado ciberestructuras que permiten el trabajo de grupos de investigación en red principalmente en áreas científicas y médicas aunque también se está utilizando en estudios sociológicos y de mercado.

El uso de las tecnologías para el aprendizaje permite obtener información sobre cómo interactúan los estudiantes con el contenido, con los materiales de aprendizaje, así como con las redes sociales que se forman, la interacción con el profesorado, con los compañeros, etc. Por ejemplo, un sistema de gestión de aprendizaje tan utilizado en las universidades como Moodle captura una gran cantidad de datos, incluyendo el tiempo dedicado a un recurso, la frecuencia de publicación, el número de inicios de sesión, los documentos leídos, la participación en los foros, etc. Estos datos son similares a lo que Google Analytics recoge en relación con el tráfico en Internet. De ahí, el nombre de learning analytics que recibe esta tendencia.

Las analíticas de aprendizaje consisten en la interpretación de un amplio rango de datos producidos y recogidos acerca de los estudiantes para orientar su progresión académica, predecir actuaciones futuras e identificar elementos problemáticos. El objetivo de la recolección, registro, análisis y presentación de estos datos es posibilitar que los profesores puedan adaptar de manera rápida y eficaz las estrategias educativas al nivel de necesidad y capacidad de cada alumno. Aun en sus primeras etapas de desarrollo, las analíticas de aprendizaje responden a menudo a la necesidad de llevar a cabo el seguimiento y control de la actividad en los campus virtuales para la toma de decisiones estratégicas. Por otro lado, pretenden también aprovechar la gran cantidad de datos producidos por los estudiantes en actividades académicas En general, la información aportada permite personalizar la acción formativa y diseñar entornos de aprendizaje acordes con las necesidades, intereses y formas de interacción de profesores y estudiantes. El registro estadístico de la actividad de estudiantes y docentes permite también identificar puntos conflictivos de un proceso de enseñanza-aprendizaje.

La aplicación de este tipo de tecnología en la investigación educativa tiene implicaciones importantes ya que se hace necesario modificar los métodos estadísticos tradicionales y además se precisan nuevas herramientas para analizar las redes sociales, los grados de conectividad, la evaluación de los atributos cualitativos de los discursos, las contribuciones de cada alumno en relación a un tema de discusión, etc.

En relación a los métodos estadísticos, Markauskaite (2011) afirma que las diferencias más importantes respecto a la estadística tradicional (tabla 1) es que los nuevos métodos de minería de datos combinan la exploración interpretativa con los datos científicos. Se necesita trabajar con los datos para poder descubrir las relaciones, los patrones de conexión y es difícil tener ideas a priori.

Retos y tendencias sobre el futuro de la investigación acerca del aprendizaje con tecnologías digitales. Begoña Gros. 
Tabla 1. Características de los métodos estadísticos. Fuente: Markauskaite (2011: 241).

\begin{tabular}{|l|l|l|}
\hline Características & Estadística & Minería de datos \\
\hline Enfoque & Inductivo & Deductivo \\
\hline Papel de la teoría & Informa las hipótesis & Informa procesos \\
\hline Ejemplos y datos & Experimentos & Reales y de conveniencia \\
\hline Proceso de indagación & De la hipótesis a la teoría & $\begin{array}{l}\text { De los datos a los patrones, } \\
\text { reglas e hipótesis }\end{array}$ \\
\hline Generalización & Explicativa & Predictiva \\
\hline Objetivo & Afirmar o negar una teoría & $\begin{array}{l}\text { Informas sobre la acción, } \\
\text { proponer nuevas teorías }\end{array}$ \\
\hline Énfasis analítico & Rigor estadístico & Rigor informativo \\
\hline
\end{tabular}

Los programas para el análisis de redes sociales también son herramientas útiles para examinar el aprendizaje en línea y ofrecen la posibilidad de identificar las intervenciones que pueden aumentar el potencial de una red para apoyar el aprendizaje de los estudiantes. Hay muchas herramientas disponibles para el análisis de redes sociales en el contexto de aprendizaje. Por ejemplo, SNAPP7 ${ }^{1}$ es un programa de visualización de libre acceso que reinterpreta envíos de los foros de discusión como un diagrama de red. Éstos también pueden ser utilizados para trazar el crecimiento de las comunidades de un curso y para identificar a los estudiantes que no se mantienen activos. Las fronteras cada vez más difusas entre el aprendizaje formal e informal lleva a que una misma persona pueda estar participando en un curso de un campus virtual, seguir una serie de twitters y blogs, comunicarse en foros con los compañeros del curso y de forma síncrona con amigos y colegas, etc. Buckingam y Ferguson (2011) señalan que el uso del rastro digital se puede aplicar a una amplia variedad de contextos y permitir analizar el comportamiento en una amplia variedad de situaciones. A corto plazo, el análisis de contexto se puede utilizar para extraer datos fácilmente disponibles, tales como la información del perfil, las marcas de tiempo, el sistema operativo y la ubicación. Estos análisis apoyan las recomendaciones que sean apropiadas para la situación de los alumnos, el tiempo que tienen disponible, los dispositivos que pueden acceder, su función actual y sus metas futuras. Análisis de contexto también se puede utilizar para resaltar la actividad de otros estudiantes en una comunidad o una red, a través de las nubes de etiquetas, las visualizaciones de datos, los flujos de actividad y etiquetasutilizadas.

Las instituciones que carecen de la infraestructura necesaria para el análisis de cálculo intensivo y los motores de recomendación tendrán que recurrir a los servicios en "nube" de la informática. Estos también serán utilizados por los alumnos individuales o comunidades que necesitan esos servicios.

Este tipo de investigación está en sus inicios y queda mucho camino por hacer. "Se precisa de importantes aportaciones de las comunidades educativas y de las políticas sociales para poder adaptar las técnicas digitales para resolver preguntas substanciales del campo educativo. Esto implica que la investigación educativa debe desplazarse de la

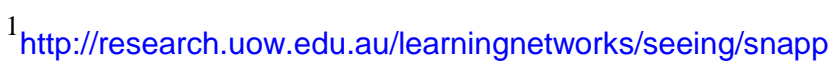

Retos y tendencias sobre el futuro de la investigación acerca del aprendizaje con tecnologías digitales. Begoña Gros. 
investigación basada en hipótesis, teóricamente fundamentada hacia modelos de exploración más abiertos, y de la tradición de publicación científica a un modelo de difusión y co-construcción del conocimiento" Markauskaite (2001: 249).

El síntesis, la finalidad de este tipo de investigación es proporcionar oportunidades para integrar datos, aplicar el conocimiento desde perspectivas diversas. Se precisan desarrollar infraestructuras que proporcionen compartir datos en repositorios digitales. Todavía hay mucho camino por recorrer ya que también nos enfrentamos a problemas éticos derivados del uso de los datos y su privacidad.

\section{Compartir y comunicar}

Para mejorar la investigación, la colaboración entre investigadores es fundamental. En este sentido, creemos que es preciso avanzar en tres aspectos: el trabajo conjunto interdisciplinar, compartir datos para que éstos sean re-utilizarlos y contribuir a la difusión abierta del conocimiento.

Olson et al. (2008) afirma que a pesar de que está demostrado que hay una correlación entre la colaboración y la productividad e impacto de los colaboradores, ésta sigue siendo muy difícil entre los académicos. No obstante, la necesidad de colaborar se hace cada día mas patente debido a que los problemas de investigación precisan enfoques más complejos. Esta realidad es muy clara en la investigación sobre el uso de las tecnologías digitales. La relación entre el diseño de las prestaciones tecnológicas y pedagógicas requiere un trabajo mucho más multi e interdisciplinarios. En este momento, el trabajo en equipo no se produce solo dentro de una determinada institución sino que los equipos son cada vez mas internacionales. Por ello, la investigacióntambién requiere de infraestructuras que le permitan compartir los datos generados a través de los diversos grupos. En otras disciplinas ya existe esta tradición pero no es fácil encontrar ejemplos en el ámbito educativo.

No sólo se trata de trabajar en grupos amplios desde el punto de vista disciplinar sino también de aunar esfuerzos y compartir datos. Todos los investigadores tenemos problemas para obtener datos en un área de estudio en que habitualmente analizamos situaciones en contextos reales. Compartir y re-utilizar datos puede ayudar a mejorar la propia investigación aportando una mayor transparencia a los procesos de obtención y análisis de los mismos. A modo de ejemplo, en los últimos he dirigido varias tesis sobre el aprendizaje colaborativo con soporte tecnológico. Y, además, he intervenido en tribunales de tesis sobre este mismo tema. Mi conclusión es que, los esfuerzos realizados por las investigadoras para obtener datos (siempre en contextos reales) y obtenidos en tiempos breves ( 1 semestre, parte de un curso) sirven para ser analizados en una sola ocasión (la tesis), bajo una única mirada y, en la mayoría de las ocasiones ya no se vuelven a utilizar. Las lecturas de cada tesis son necesariamente parciales, por lo que tenemos como resultado muchos trabajos que, o bien se repiten o analizan un aspecto muy concreta de la colaboración (interacción del grupo, papel ). Si pudiéramos analizar los datos desde diversas ópticas, ver resultados a lo largo del tiempo, obtendríamos mejores resultados.

Retos y tendencias sobre el futuro de la investigación acerca del aprendizaje con tecnologías digitales. Begoña Gros. 
Los modelos tradicionales de edición y comunicación académica también están en permanente cambio. La difusión de los resultados es inmediata y este hecho ha afectado de forma notable a la propia producción del conocimiento. Como afirma Alonso et al. (2008), uno de los grandes logros de movimiento para el libre acceso a las publicaciones científicas ha sido el apoyo institucional recibido a través de organizaciones nacionales y supranacionales. Además, se trata de una iniciativa organizada en la que están implicados tanto centros de investigación (universidades, institutos, instituciones...) gestores de información (bibliotecas, centros de información...) como los propios investigadores.

Actualmente, la mayoría de las universidades disponen de repositorios institucionales que permiten distribuir la creación científica, incluyendo informes científicos y técnicos, informes de trabajo, pre-prints, artículos y datos. Tener un repositorio supone una ventaja competitiva para la propia organización ya que le proporciona un valor añadido al incrementar la visibilidad y el impacto de sus investigadores.

El acceso abierto también se ha convertido en un requisito de muchas agencias públicas de financiación de manera que se permita el acceso a las publicaciones y los datos que derivan de los proyectos que financian.

Por último, el uso de la web social también favorece la investigación. Las aplicaciones de la web social a la investigación se pueden agrupar en tres grandes bloques: compartir la investigación, compartir los recursos y compartir los resultados. "En el primer grupo, se integran las tecnologías que permiten compartir el desarrollo de una investigación, ya se trate de redes sociales específicas, bases de datos de científicos donde los perfiles profesionales se relacionan, plataformas diseñadas para la investigación compartida o servicios de apoyo para los procesos de una investigación. En el segundo, se agrupan todas las herramientas que permiten a los investigadores compartir los recursos de información que emplean: referencias bibliográficas, favoritos web o índices de citas. Por último, existe un tercer grupo de aplicaciones que tienen como denominador común las posibilidades de difundir de forma abierta los resultados de una investigación. Además de los depósitos en abierto, se trata de servicios de promoción de información basados en blogs y wikis, con amplia capacidad de redifusión, gracias a las tecnologías RSS" (REBIUN, 2010: 3).

\section{Conclusiones}

La investigación sobre el uso de las TICs en la educación constituye un ámbito consolidado de estudio que todavía tiene algunas limitaciones. Uno de los principales problemas es que encontramos muchas investigaciones centradas en el análisis de las "bondades" de una determinada tecnología. Consideramos que es necesario superar una aproximación simplista e incorporar preguntas que orienten la investigación y permitan comprender la interacción dinámica entre el aprendizaje y las prestaciones tecnológicas. Esta aproximación obliga a avanzar en la utilización de nuevos métodos e instrumentos de investigación.

Retos y tendencias sobre el futuro de la investigación acerca del aprendizaje con tecnologías digitales. Begoña Gros. 
Hemos introducido dos aproximaciones diferentes pero que consideramos prometedoras: la investigación basada en el diseño y el uso de los datos producidos en la red.

La investigación basada en el diseño se lleva a cabo en entornos de aprendizaje reales y complejos y puede aportar información relevante en la propia investigación sobre el diseño de entornos de aprendizaje con soporte tecnológico. La aplicación de esta metodología tiene como tarea pendiente mejorar la propia técnica de diseño para facilitar la transferencia de los resultados

El uso de Internet para la comunicación y el aprendizaje genera una amplia gama de datos que nos permiten obtener información mucho más masiva y nos enfrentan al reto de investigar con planteamientos deductivos. Es este un enfoque muy prometedor que puede dar respuesta tanto a análisis macro de condiciones sociales y políticas como a estudios micro que permitan conocer comportamientos individuales de los aprendices e incrementar la calidad del apoyo y la retroalimentación.

El uso de Internet no sólo tiene efectos en la obtención de datos sino también en la difusión del conocimiento. La aplicación de las tecnologías y las redes de la información posibilita que los resultados de la investigación puedan estar a disposición del resto de la comunidad científica de manera universal e inmediata. El acceso abierto a los datos y a los resultados de la investigación modifica la propia investigación que necesariamente pasa a ser una producción grupal y colaborativa.

Presentación del artículo en versión original: 20 de septiembre de 2012

Fecha de aprobación: 25 de septiembre de 2012

Fecha de publicación: 15 de julio de 2016

Gros, B. (2016). Retos y tendencias sobre el futuro de la investigación acerca del aprendizaje con tecnologías digitales. RED. Revista de Educación a Distancia. 50(10). Consultado el (dd/mm/aaaa) en http://www.um.es/ead/red/50

\section{Referencias}

Alonso, J, Subirats, I \& Martínez Conde, M.L. (2008). Informe APEI sobre acceso abierto. Gijón: Asociación Profesional de Especialistas en Información.

Baker, R. S. J. D., \&Yacef, K. (2009). The state of educational data mining in 2009: a review and future visions. Journal of Educational Data Mining, 1(1), 3-17.

Brown, A. L. (1992). Design experiments: Theoretical and methodological challenges in creating complex interventions in classroom settings. Journal of The Learning Sciences, 2(2), 141-178.

Buckingham Shum, S. and Ferguson, R. (2011). Social Learning Analytics. Technical Report KMI-11-01, Knowledge Media Institute, The Open University, UK. Disponiblehttp://kmi.open.ac.uk/publications/pdf/kmi-11-01.pdf

Retos y tendencias sobre el futuro de la investigación acerca del aprendizaje con tecnologías digitales. Begoña Gros.

Pg. 11 de 13 
Cabero, J. et al. (2009). E-learning: Meta-análisis de investigaciones y resultadosalcanzados. [tecnologiaedu.us.es/nweb/htm/pdf/ProyectoEA08.pdf]

Chinn,C.,Erkens,G. \&Puntambekar, S. (Eds.). (2007). CSCL 2007. The Computer Supported Collaborative Conference. Rutgers: International Society of the Learning Sciences.

Collins, A. (1992). Toward a design science of education. In E. Scanlon\&T. O’Shea (Eds.), New directions in educational technology (pp. 15-22). New York: Springer-Verlag.

Cuban, L. (2001). Oversold and underused: Computers in the classroom. Cambridge, MA: Harvard University Press.

Goodyear,P. (2011). Emerging Methodological Challenges for Educational Research. En L. Markauskaite.,P.Freebody., J. Irwin (Eds). (2011). Methodological Choice and Design: London/NY: Springer, 253-267.

Hung, J (2012). Trends of e-learning research from 2000 to 2008: Use of text mining and bibliometrics, British Journal of Educational Technology. 2012;43(1):5-16.

Markauskaite, L. (2011). Digital Knowledge and Digitl Research: What does eResearch Offer Education and Social Policy?. En L. Markauskaite.,P.Freebody., J. Irwin (Eds). (2011). Methodological Choice and Design: London/NY: Springer, 235-252.

Olson, G. M., Zimmerman, A., \&Bos, N. (2008). Scientific collaboration on the internet. Cambridge, MA: The MIT Press.

Plomp, T. \&Nieveen, N. (2009). Introduction to educational design research.Enschede, The Netherlands: SLO Netherlands Institute for Curriculum Development.

REBIUN (2010).Ciencia 2.0: aplicación de la web social a la investigación. Madrid. Disponible en: eprints.rclis.org/bitstream/10760/3867/1/Ciencia20_rebiun.pdf

Reeves, T (2011). Can Educational Research Be Both Rigorous and Relevant?.Journal of the International Society for Design and Development in Education, March, 4.

Reeves, T. C. (2006). How do you know they are learning? The importance of alignment in higher education. International Journal of Learning Technology, 2(4), 294-309.

Reimann, P (2011). Design Research. En L.Markauskaite, P.Freebody, J. Irwin (eds).Methodological Choice and Design. Springer, 37-56.

Reimann, P. (2011). Desig Based Research. En L. Markauskaite.,P.Freebody., J. Irwin (Eds). (2011). Methodological Choice and Design: London/NY: Springer, 37-50.

Rowland, G (2008). Design and research: Partners for educational innovation. Educational Technology, 48 (6), 3-9.

Schoenfeld, A. H. (2009). Bridging the cultures of educational research and design. EducationalDesigner, 1(2). Disponible en http://www.educationaldesigner.org/ed/volume1/issue2/article5

Stoker, G- John, P (2009). "Design Experiments: Engaging Policy Makers in the Search for Evidence abputAhat Works". Political Studies, 57, 356-373.

The Design-Based Research Collective (2003). Design-Based research: An Emerging Paradigm for Educational Inquiry. Educational Researcher, Vo. 32, No. 1, 5-8

Retos y tendencias sobre el futuro de la investigación acerca del aprendizaje con tecnologías digitales. Begoña Gros.

Pg. 12 de 13 
Van den Akker, J. (1999). Principles and methods of development research. In van den Akker, J., Nieveen, N., Branch, R. M., Gustafson, K. L. \&Plomp, T. (Eds.), Design methodology and developmental research in education and training (pp. 1-14). The Netherlands: Kluwer Academic Publishers.

Zawacki-Richter, O. (2009). Research Areas in Distance Education: A Delphi Study. International Review of Research in Open and Distance Learning, 9 (10): 1-17.

Zawacki-Richter, O. et al. (2009). Review of Distance Education Research (2000 to 2008): Analysis of Research Areas, Methods and Authorship Patterns. International Review of Research in Open and Distance Learning, 6 (10): 21-49 